\title{
N-(2-Acyl 1,3-indanedione) Triethyl Ammonium Iodide, a New Growth Promotor
}

In recent years there is an increasing tendency to prepare water-soluble, biologically active organic nuclei. The solubilizing groups are compounds containing $-\mathrm{OH},-\mathrm{COOH}$, $-\mathrm{SO}_{2} \mathrm{OH}$ and quaternary ammonium radicle. The chemical 2-acyl 1, 3-indane dione has been used as blood coagulant ${ }^{1}$. Another derivative, 2-(2-alkylphenyl)-1,3-indane dione, is known to possess anti-coagulating, anti-inflammatory, muticidal and herbicidal properties ${ }^{2}$. The authors have studied the effect of a new derivative, $\mathrm{N}$-(2-acyl 1,3-dione) triethyl ammonium iodide, on growth behaviour of the seedlings of Cyamopsis tetragonoloba where the compound showed its growth-regulating property by enhancing the growth. The authors also studied the interaction of $\mathrm{N}$-(2-acyl 1,3-indanedione) triethyl ammonium iodide (L-TEAI) and N-Benzyl orthofluoro phenoxy acetamide (NBOFPA), a growth retardant ${ }^{3}$.

The nucleus, 2-acyl 1,3-indane dione, was prepared by reaction of dimethyl phthalate and acetone in the presence of sodium methoxide by the method of KrLGORE et $\mathrm{al}^{4}$. The chemical N-(2-acyl 1,3-indane dione) triethyl ammonium iodide was obtained by King's ketoalkylation

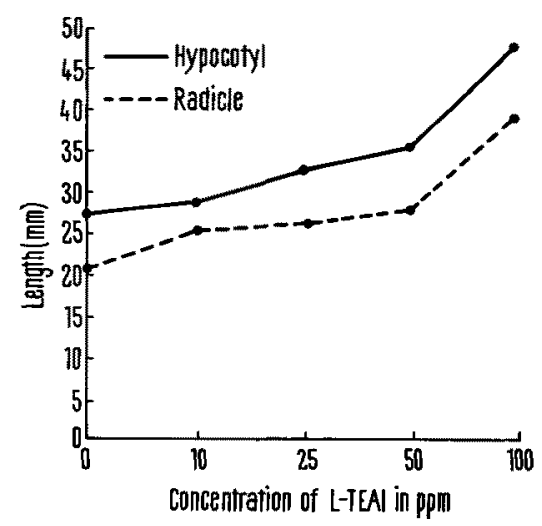

Fig. 1. Influence of different concentrations of L-TEAI on the growth of Cyamopsis tetragonoloba seedlings after $96 \mathrm{~h}$ of germination.

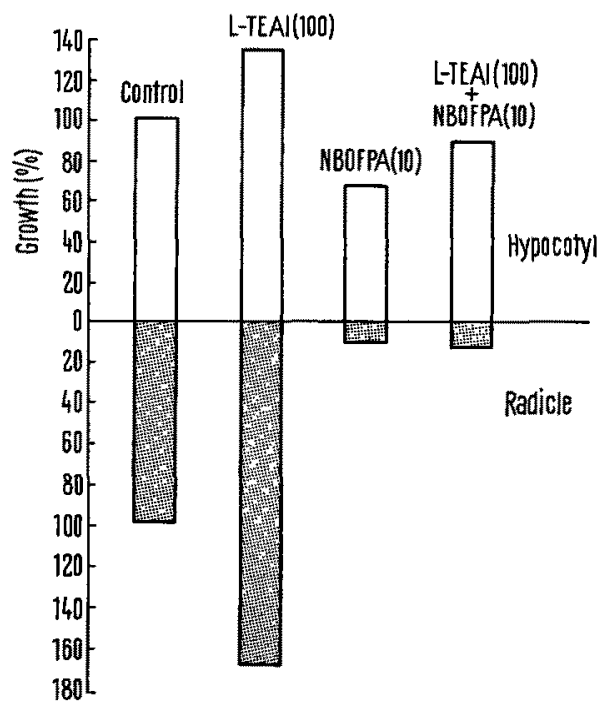

Fig. 2. Reversal of L-TEAI induced hypocotyl and radicle growth by N-benzyl orthofluorophenoxy acetamide. reaction. This chemical was solubilized by introduction of triethyl ammonium iodide group. The compound is a brown crystalline solid, highly soluble in water, having mp 131-132 ${ }^{\circ} \mathrm{C}$ with following structural and molecular formula:<smiles>C[N+](C)(C)NOP(=O)([I-])[N+](C)(C)C</smiles>

The seeds of Cyamopsis tetragonoloba were soaked in different concentrations of L-TEAI for $6 \mathrm{~h}$ at room temperature, while control seeds were soaked in distilled water for the same period. To study the interaction of this chemical with NBOFPA, the seeds were soaked in a solution containing $100 \mathrm{ppm}$ and $10 \mathrm{ppm}$ of the chemicals respectively. After soaking the seeds for $6 \mathrm{~h}$, seeds were washed with water and were allowed to germinate in petri dishes lined with moist filter paper in an incubator at $30 \pm 1{ }^{\circ} \mathrm{C}$. The length of radicle and hypocotyl was continuously recorded after every $24 \mathrm{~h}$ of germination upto $120 \mathrm{~h} .3$ replicates for germination were taken. Length of 10 seedlings was recorded for every replicate. The experiments were carried out in complete absence of light.

The indane derivative studied clearly indicated a growth-promoting activity both for radicle and hypocotyl (Figure 1). The promotion of growth was linear with concentration of the chemical used upto $200 \mathrm{ppm}$. Growth of the hypocotyl was promoted by $72.5 \%$ and that of radicle by $85.5 \%$ respectively after $96 \mathrm{~h}$ of germination. But when L-TEAI (100 ppm) was used along with NBOFPA (10 ppm) it failed to bring any promotion in growth (Figure 2). It has been reported that gibberellic acid reversed the NBOFPA-induced inhibition ${ }^{5}$. But L-TEAI completely failed to reverse either the hypocotyl or radicle inhibition induced by NBOFPA. It is evident that the growth-promoting property is completely different from that of gibberellic acid. It appears probable that growth-promoting mechanism of L-TEAI may be one and the same for radicle and hypocotyl, because it neither indicated any interaction with NBOFPA for radicle and hypocotyl elongation, nor did it show any differential growth for radicle and hypocotyl when treated with NBOFPA in mixture.

Zusammenfassung. I-TEAI scheint einen wuchsstoffähnlichen Charakter zu haben, der sich von demjenigen der Gibberelline unterscheidet.

K. L. Menaria, S. Kathju and M. N. TEWARI

Laboratory of Organic Chemistry,

Department of Chemistry, and

Laboratory of Tissue Culture and Biochemistry, Department of Botany, The University, Jodhpur (India), 5 June 1970.

1 E. A. Shafro, Chem. Abstr. 70, 2292 (1969).

2 B. Vorhen, Chem. Abstr. 69, 58991 (1968).

s M. N. TewarI, Experientia 25, 433 (1969).

4. B. Kulgore, J. H. Ford and W. C. Wolfe, Ind. Engng. Chem. 34,494 (1942).

* S. Kath Ju and M. N. Tewari, Curr. Sci. 39, 46 (1970). 